\title{
Umgang mit Patientenverfügungen in Deutschland
}

\section{Sichtweisen leitender Intensivmediziner}

\author{
S. Langer ${ }^{1}$, I. Stengel ${ }^{1}$, S. Fleischer ${ }^{1}$, R. Stuttmann ${ }^{2}$, A. Berg ${ }^{1}$
}

Hintergrund und Fragestellung I Auch sechs Jahre nach dem im Jahr 2009 in Kraft getretenen „Dritten Gesetz zur Änderung des Betreuungsrechts“ wird die Patientenverfügung weiterhin kontrovers diskutiert. Die vorliegende Studie zeigt erstmals deutschlandweit die Sichtweisen leitender Intensivmediziner zur Umsetzung von Patientenverfügungen sowie ihre Erfahrungen zu diesbezüglichen ethischen Herausforderungen.

Methode I Es erfolgte eine standardisierte, schriftliche Befragung aller deutschen Kliniken mit mehr als 300 Betten und einer anästhesiologisch geführten Intensivstation mittels eines auf den Ergebnissen einer qualitativen Vorstudie basierenden Fragebogens. Dieser richtete sich an einen leitenden Intensivmediziner pro Klinik.

Ergebnisse I Der Fragebogen wurde von 222/299 Ärzten beantwortet (Rücklaufquote: 74,2\%). Trotz einer Vielzahl geäußerter Probleme im Um- gang mit Patientenverfügungen wird diese von $70,6 \%(n=154 / 218)$ der Intensivmediziner generell als hilfreich empfunden. 86,5\% ( $n=185 / 214)$ der Befragten wünschen sich mehr therapiezielorientierte als maßnahmenorientierte Patientenverfügungen. Zudem plädieren 95,9\% ( $n=207 / 216)$ für eine qualifizierte fachliche Beratung zu intensivmedizinischen Inhalten. In Bezug auf einen Behandlungsabbruch ist eine Akzeptanz des Selbstbestimmungsrechtes des Patienten sichtbar. Finale Extubation gemäß dem Patientenwillen wird in $79,4 \%(n=173 / 218)$ der Kliniken durchgeführt; den Vorgang der finalen Extubation erleben 54,8\% ( $n=113 / 206)$ der Ärzte als belastend.

Diskussion | Zukünftig sind geeignete unterstützende Konzepte und Maßnahmen zur Erstellung aussagekräftiger Patientenverfügungen und $\mathrm{zu}$ einer wirksamen Umsetzung im intensivmedizinischen Alltag notwendig.

\section{Einleitung}

Mit dem im Jahr 2009 in Kraft getretenen Dritten Gesetz zur Änderung des Betreuungsrechts, dem sogenannten „Patientenverfügungsgesetz“, wurde das Vorsorgeinstrument „Patientenverfügung“ (PV) gesetzlich gestärkt. Die fachliche und gesellschaftliche Diskussion um ihre Vor- und Nachteile sowie ihre praktische Umsetzung wird auch weiterhin kontrovers geführt, jedoch wächst parallel beständig der Anteil der Patienten in Krankenhäusern, die eine PV erstellt haben [1].

Gerade im intensivmedizinischen Bereich ergeben sich dabei besondere Anforderungen an das Behandlungsteam [2]. Kürzlich wurde die Aussagekraft von PV auf vier Intensivstationen eines Universitätsklinikums analysiert. Im Zeitraum zwischen September 2013 und März 2014 hatten $11,8 \%(n=112 / 950)$ der Intensivpatienten mit einer Liegedauer über 48 Stunden eine PV verfasst [3]. Bisher liegen in diesem Setting jedoch nur wenig nationale, empirische Daten zum Umgang mit PV und zur ärztlichen Handlungspraxis am Lebensende vor [3-7]. Den Fokus der vorliegenden Studie bildeten daher die Sichtweisen von leitenden Intensivmedizinern zur Umsetzung von PV sowie ihre Erfah- rungen zu diesbezüglichen ethischen Herausforderungen.

\section{Methode}

Die Untersuchung erfolgte als standardisierte, postalische Befragung aller deutschen Kliniken mit mehr als 300 Betten und einer anästhesiologisch geführten Intensivstation. Zur Identifikation der Kliniken $(n=299)$ wurde das Deutsche Krankenhausverzeichnis (http://www.deutscheskrankenhaus-verzeichnis.de) genutzt. Der Fragebogen sollte von einem leitenden Intensivmediziner pro Klinik ausgefüllt werden, das heißt die Teilnehmer sollten Entscheidungsträger auf der anästhesiologisch geführten Intensivstation (Chefarzt, leitender Arzt, Oberarzt) und Facharzt für Anästhesie und Intensivmedizin sein. Die Datenerhebung erfolgte von Januar bis Mai 2015; die Teilnahme an der Befragung war freiwillig. Zur Erhöhung der Rücklaufquote wurde die TotalDesign-Methode [8] mit mehreren Kontaktaufnahmen und Erinnerungen (schriftlich, telefonisch sowie per E-Mail) angewendet.

Die theoretische Grundlage zur Entwicklung des Fragebogens bildete eine eigene Vorstudie [9], in
Institute

1 Martin-Luther-Universität Halle-Wittenberg, Medizinische Fakultät, Institut für Gesundheitsund Pflegewissenschaft

2 Berufsgenossenschaftliches Klinikum Bergmannstrost Halle, Klinik für Anästhesiologie und Intensivmedizin

\section{Korrespondenz}

Susan Langer, M.Sc. GPW Institut für Gesundheits- und Pflegewissenschaft Medizinische Fakultät Martin-Luther-Universität Halle-Wittenberg Magdeburger Straße 8 06112 Halle (Saale) susanlanger@gmx.de Tel: 0345-5574466

Dr. rer. medic. Almuth Berg Institut für Gesundheits- und Pflegewissenschaft Medizinische Fakultät Martin-Luther-Universität Halle-Wittenberg Magdeburger Straße 8 06112 Halle (Saale) almuth.berg@medizin. uni-halle.de Tel: 0345-5574132 
Tab. 1 Merkmale der Studienteilnehmer. der die Probleme von Intensivmedizinern im Umgang mit PV anhand eines qualitativen Designs untersucht wurden. Der Fragebogen beruht auf den dort generierten Themenbereichen [9] und umfasst 116 einzeln zu beantwortende Items. Bei den Antworten handelte es sich mehrheitlich um 4-stufige verbalisierte Skalen (z.B.: stimme gar nicht zu - stimme eher nicht zu - stimme eher zu - stimme voll zu). Vorab wurde der Fragebogen einem Zwei-Phasen-Pretest [10] unterzogen.

Für die Datenauswertung wurde die Software SPSS 22 genutzt. Da es sich bei der Zielstellung der Erhebung um eine Beschreibung und Darstellung der Sichtweisen leitender Intensivmediziner handelt, wurden zur Auswertung der Daten ausschließlich Methoden der deskriptiven Statistik angewendet und die Antwortskalen dichotomisiert. Die dargestellten Prozentangaben sind Anteile der jeweils gültigen Werte, wobei sich rundungsbedingte Abweichungen von $100 \%$ in den Summen ergeben können.
Um das Antwortverhalten der Teilnehmer näher einschätzen zu können, wurde zudem eine NonResponder-Analyse in Bezug auf Trägerschaft der Institution und Bundesland durchgeführt.

\section{Ergebnisse}

Bundesweit beantworteten 222 von 299 kontaktierten Kliniken den Fragebogen, was einer Rücklaufquote von $74,2 \%$ entspricht. Die Non-Responder-Analyse zeigte keine Assoziation zwischen der Trägerschaft der Einrichtung oder dem Bundesland und der Teilnahmebereitschaft.

Die Stichprobe setzte sich zusammen aus $(\triangleright$ Tab. 1)

- Chefärzten,

- Leitenden Ärzten,

- Oberärzten und

- Fachärzten.

\section{Variable (gültige Werte, $n$ )}

Art der Einrichtung $(\mathrm{n}=\mathbf{2 2 2})$

Klinik der Schwerpunkt- und Maximalversorgung

davon konfessioneller Träger

Klinik der Grund- und Regelversorgung davon konfessioneller Träger

Universitätsklinik

Position ( $\mathrm{n}=\mathbf{2 2 2}$ )

Chefarzt

Leitender Arzt

Oberarzt

Facharzt

3

Größe der Intensivstation ( $\mathrm{n}=197$ )

$<10$ Betten

19

10-15 Betten

80

16-20 Betten

52

21-25 Betten

$>25$ Betten

Geschlecht ( $=$ = 222)

männlich

weiblich

Alter ( $=$ 222)

$<45$ Jahre

46-55 Jahre

56-65 Jahre

$>65$ Jahre
30

39

109

58

absolute Häufigkeit, n

relative Häufigkeit, (\%)

08

26

84

24

0

1,4

9,6

40,6

26,4

8,6

14,7

17,6

24,3

49,1

26,1 


\begin{tabular}{|c|c|c|}
\hline Variable (gültige Werte, n) & absolute Häufigkeit, n & relative Häufigkeit, (\%) \\
\hline \multicolumn{3}{|c|}{ Religionszugehörigkeit (n=218) } \\
\hline evangelisch & 82 & 37,6 \\
\hline katholisch & 73 & 33,5 \\
\hline sonstige & 1 & 0,5 \\
\hline keine & 62 & 28,4 \\
\hline \multicolumn{3}{|c|}{ Konfrontation mit Patientenverfügungen $(n=221)$} \\
\hline ein- bis dreimal im Monat & 38 & 17,2 \\
\hline einmal in der Woche & 32 & 14,5 \\
\hline mehrmals pro Woche & 125 & 56,6 \\
\hline (mehrmals) täglich & 26 & 11,8 \\
\hline \multicolumn{3}{|c|}{ persönliches Vorsorgeinstrument ( $\mathbf{n = 2 2 1 )}$} \\
\hline nein & 102 & 46,2 \\
\hline ja & 119 & 53,8 \\
\hline \multicolumn{3}{|c|}{ Wenn ja, welches? (Mehrfachantworten möglich; $n=119$ ) } \\
\hline Vorsorgevollmacht & 104 & 87,4 \\
\hline Patientenverfügung & 73 & 61,3 \\
\hline Betreuungsverfügung & 23 & 19,3 \\
\hline andere & 12 & 10,1 \\
\hline
\end{tabular}

Tab. 1 Fortsetzung.

Chefärzte bilden dabei mit 52,7\% ( $n=117 / 222)$ den größten Anteil. Die Größe der Intensivstationen lag zu 67,0\% ( $\mathrm{n}=132 / 197)$ zwischen 10 und 20 Betten. Von $95,0 \%(n=211 / 222)$ der Ärzte werden Patienten der Allgemeinchirurgie behandelt, zudem Patienten der Unfallchirurgie (86,0\%; $\mathrm{n}=191 / 222)$ und internistische Patienten (76,6\%; $\mathrm{n}=170 / 222$ ). Das Durchschnittsalter der Patienten schätzten $83,0 \%$ der Ärzte $(n=171 / 206)$ als $>60$ Jahre ein. $82,8 \%(n=183 / 221)$ der Ärzte wurden mindestens einmal pro Woche mit einer PV konfrontiert.

Umgang mit Patientenverfügungen | 56,6\% $(n=125 / 221)$ der Intensivmediziner sehen oft bis immer generelle Probleme im Umgang mit Patientenverfügungen. Inwieweit PV prinzipiell ihr Ziel im Rahmen der intensivmedizinischen Behandlung erfüllt, beurteilten 54,5\% ( $n=121 / 222)$ mit selten bis nie.

Gründe, eine PV zu erstellen, sind nach Meinung der Ärzte

- das Vermeiden von unnötigen Qualen und Leid (97,2\%; $n=212 / 218)$,

- die Angst vor Übertherapie (77,9\%; n=169/217) sowie

- die Entlastung von Angehörigen (73,5\%; $\mathrm{n}=150 / 204)$.

Wenn Patienten eine Meinung in der PV darlegen, diese aber im Krankheitsfall ändern, so geschieht dies nach Meinung der Ärzte weil
- sich die Ansprüche der Patienten verändern $(65,1 \% ; n=127 / 195)$,

- im Vorfeld Sachverhalte bei der Erstellung der PV unklar waren $(58,1 \% ; n=108 / 186)$ und

- Menschen mehr ertragen können, als sie sich vorgestellt haben $(53,6 \% ; n=103 / 192)$.

Die Mehrheit der Intensivmediziner war der Ansicht, dass es Formulierungsprobleme bei den Patientenverfügungen gibt, insbesondere:

- PV oft bis immer zu allgemein formuliert sind (93,2\%; $n=207 / 222)$,

- PV selten bis nie klar definiert sind (91,9\%; $\mathrm{n}=204 / 222$ ),

- PV selten bis nie den konkreten Krankheitsfall abdecken (94,6\%; $\mathrm{n}=208 / 220)$.

Nach Angaben von 78,9\% $(n=172 / 218)$ der Befragten wurden Mängel in den Formulierungen oft erst sichtbar, wenn versucht wurde, die PV umzusetzen. 70,4\% ( $n=133 / 189)$ der Teilnehmer äußern, dass bisher meist weniger als die Hälfte der ihnen vorgelegten PV ohne Probleme umsetzbar waren. 80,4\% ( $n=168 / 209)$ der Intensivmediziner sehen sich selten bis nie in der Lage, die Aussage „lebenswertes Leben“ für einen ihnen nicht bekannten Patienten beurteilen zu können. 72,1\% ( $n=158 / 219)$ der Intensivmediziner müssen oft oder immer Patienten beziehungsweise Angehörige über die Inhalte und Konsequenzen der PV erst aufklären. Dennoch sind 70,6\% ( $\mathrm{n}=154 / 218)$ der Ärzte der Ansicht, dass eine unklar formulierte PV trotzdem hilfreich sein kann. Auch empfin- 
Beurteilung von Maßnahmen, die einem Therapieabbruch entsprechen Ist für mich persönlich ...

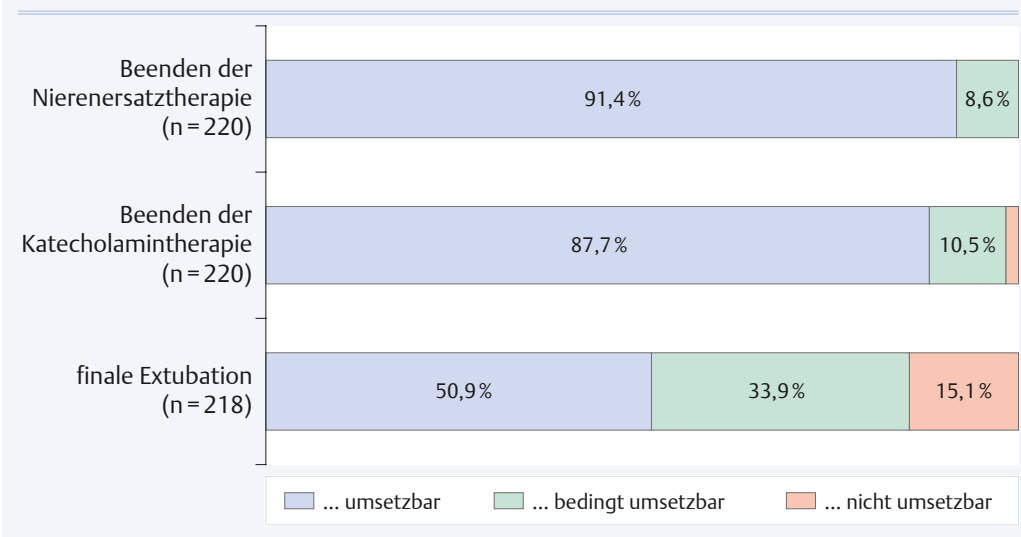

Abb. 1 Gewichtung medizinischer Maßnahmen, die einem Therapieabbruch entsprechen.

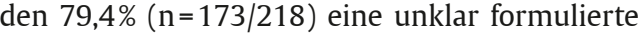
PV im Behandlungsprozess nicht als hinderlich.

Bezüglich des Zeitmanagements in Notfallsituationen sind sich die Intensivmediziner weitestgehend einig, dass in lebensbedrohlichen Situationen wie einer kardiopulmonalen Reanimation, bei einem Verkehrsunfall oder im Schockraum selten bis nie ausreichend Zeit ist,

- um nach einer PV zu fragen (75,3\%; $\mathrm{n}=165 / 219$ ),

- um sie zu lesen (89,0\%; $n=195 / 219)$,

- um sie auszulegen (92,7\%; $\mathrm{n}=202 / 218)$ oder

- um sie anzuwenden $(87,0 \% ; \mathrm{n}=187 / 215)$.

Das Konzept der gesundheitlichen Vorausplanung (Advance Care Planning, ACP) ist 36,1\% ( $n=79 / 219)$ der befragten Ärzte bekannt, bereits angewendet wird es von 11,3\% $(n=24 / 212)$.

Ethische Herausforderungen I Laut Rechtsprechung ist als Sterbehilfe das Unterlassen, Begrenzen oder Beenden lebenserhaltender medizinischer Therapiemaßnahmen (Behandlungsabbruch durch Unterlassen oder durch aktives Tun) gerechtfertigt, wenn dies dem tatsächlichen oder mutmaßlichen Patientenwillen entspricht [11]. Für $83,4 \%(n=181 / 217)$ der Intensivmediziner besteht zwischen dem Ausstellen des Beatmungsgerätes auf Patientenwunsch und aktiver Sterbehilfe (Tötung auf Verlangen) ein deutlicher Unterschied. Dass jedoch beides oft verwechselt wird, bestätigen 78,6\% ( $n=158 / 201)$ der Befragten. $69,9 \%(n=153 / 219)$ vermuten, dass es bei aktivem Tätigwerden - beispielsweise beim Ausstellen des Beatmungsgerätes oder bei der finalen Extubation - unter den Kollegen zu Unsicherheiten bezüglich der Rechtslage kommt.

Allerdings empfinden auch 64,4\% ( $n=141 / 219)$ der Intensivmediziner einen Unterschied zwischen einem widerrufbaren und einem unwiderrufbaren Therapieabbruch (zum Beispiel: Beenden der Dialyse versus finale Extubation). 30,3\% $(n=66 / 218)$ stehen mit einem unwiderrufbaren
Therapieabbruch in Konflikt, weil durch die Handlung der Tod unmittelbar eintritt. Insgesamt wird in $79,4 \%(n=173 / 218)$ der Kliniken die finale Extubation gemäß Patientenwillen durchgeführt; dementsprechend führen sie 20,6\% $(n=45 / 218)$ der Kliniken nicht durch. Den Vorgang der finalen Extubation erleben dabei 54,8\% $(n=113 / 206)$ der Intensivmediziner oft oder immer als belastend.

Die Problematik, inwiefern ein Therapieabbruch für die Ärzte persönlich umsetzbar ist, sollte an drei Beispielen gewichtet werden ( $\bullet$ Abb. 1). Weiterhin wurde anhand einer Fallvignette nach dem persönlichen Umgang mit einem Therapieabbruch gefragt $(\triangleright$ Abb. 2 ).

87,3\% ( $n=192 / 220)$ der Ärzte stimmen zu, dass es Intensivmedizinern schwerer fällt, eine Therapiebegrenzung oder einen Therapieabbruch bei Patienten vorzunehmen, wenn die Patienten an einer nicht unmittelbar zum Tode führenden $\mathrm{Er}$ krankung leiden. Wenn das Moralempfinden der Gesellschaft kongruent ist, beispielsweise bei Tumorleiden im Endstadium oder bei Amyotropher Lateralsklerose, fällt es Intensivmedizinern hingegen leichter. Diese Aussage wiederum wird von 95,9\% ( $n=209 / 218)$ der Befragten bestätigt.

Obwohl sich 96,2\% ( $n=202 / 210)$ der Befragten in der Regel mit dem Patientenwillen identifizieren konnten und ebenfalls 96,2\% ( $n=205 / 213)$ sich bisher auch mit dem Patienten oder Vertreter einigen konnten, gaben $32,0 \%(n=70 / 219)$ der Intensivmediziner an, dass sie den Patientenwillen bereits gegen ihre ärztliche Überzeugung umsetzen mussten. Bei 53,0\% ( $n=116 / 219)$ der Befragten kam es mindestens einmal während ihrer Berufsjahre vor, dass eine Einschätzung des Betreuungsgerichtes notwendig wurde, da sich mit dem Stellvertreter kein Einvernehmen zum Patientenwillen erreichen ließ. Bei $11,4 \%(n=25 / 219)$ traf dies mindestens schon viermal zu.

Umsetzung von Patientenverfügungen im intensivmedizinischen Kontext I Insgesamt vertreten über $90 \%$ der Teilnehmer die Meinung, dass der medizinische Laie keine realistische Vorstellung von der Intensivmedizin (95,5\%, $n=210 / 220)$ und falsche Vorstellungen von den aktuellen diagnostischen und therapeutischen Möglichkeiten $(92,3 \%, n=203 / 220)$ hat. Auch sind sich die Ärzte überwiegend einig, dass Angehörige die Situation des Patienten oft nicht einschätzen können $(90,8 \% ; n=198 / 218)$ und mit der Situation überfordert sind (96,8\%; $n=213 / 220)$. Ebenso gehen $78,1 \%(n=164 / 210)$ davon aus, dass die Szenarien auf einer Intensivstation für Mediziner anderer Fachbereiche oft schwer beurteilbar sind.

Infolge dessen halten $87,7 \%$ der Befragten $(n=192 / 219)$ eine Beratung zu intensivmedizini- 
schen Inhalten der PV durch Ärzte für notwendig und 91,7\% ( $n=199 / 217)$ stimmen einer Beratung ohne ärztliche Beteiligung nicht zu. Zudem möchten $88,2 \%(n=194 / 220)$ der Ärzte, dass eine solche Beratung durch Intensivmediziner stattfindet.

Generelle Einstellungen zur Patientenverfügung I Als Vorteil einer PV sehen 97,3\% ( $n=215 / 221)$ der Teilnehmer, dass sich die Menschen durch die Erstellung der PV mit dem Tod und ihren Vorstellungen zum Lebensende beschäftigen. Weitere Vorteile bestehen für 94,5\% ( $n=208 / 220)$ in einer Hilfe für Angehörige, wenn diese mit der Situation der Einwilligungsunfähigkeit des Patienten überfordert sind. Ebenfalls positiv finden $86,8 \%$ ( $n=190 / 219$ ), dass Bevollmächtigte oder Betreuer keine Entscheidungen über die Fortführung von Therapien treffen müssen, wenn der Patient diese bereits getroffen hat.

23,3\% ( $n=47 / 202)$ der Intensivmediziner können sich vorstellen, dass unter Zeitdruck eine PV auch eine Gefahr für den Patienten darstellen kann, weil diese umgesetzt werden könnte, ohne zu eruieren, ob der geäußerte Patientenwille dem tatsächlichen Patientenwillen entspricht. Weiterhin sehen $20,1 \%(n=42 / 209)$ eine mögliche Gefahr darin, dass die PV von Ärzten in Akutsituationen nur oberflächlich gelesen und eine voreilige Entscheidung getroffen werden könnte.

In Bezug auf das „Patientenverfügungsgesetz“ empfinden 69,4\% ( $n=145 / 209$ ) der Intensivmediziner mehr Spielraum, um den Patientenwillen umzusetzen. Laut 80,7\% ( $n=176 / 218)$ der Teilnehmer hat bei den Ärzten bereits ein Umdenken zum Selbstbestimmungsrecht des Patienten stattgefunden. In der neuen Rechtslage durch das „Patientenverfügungsgesetz“ sehen darüber hinaus 58,2\% $(n=125 / 215)$ der Befragten mehr Sicherheit für Ärzte und 47,3\% ( $n=98 / 207)$ eine Minimierung der Sorge um unterlassene Hilfeleistung.

Entsprechend der öffentlichen Diskussion wurde ebenfalls ein Meinungsbild zum Thema Gesetzesänderungen zum ärztlich assistierten Suizid
Fallvignette:

Ein 60-jähriger Patient erleidet durch einen Unfall eine hohe Querschnittslähmung. Er ist wach, ansprechbar, kooperativ und verlangt das Ausstellen des Beatmungsgeräts. Nach Ethikberatungen und einem psychiatrischen Konsil wird bestätigt, dass sein Wille umgesetzt werden müsste.

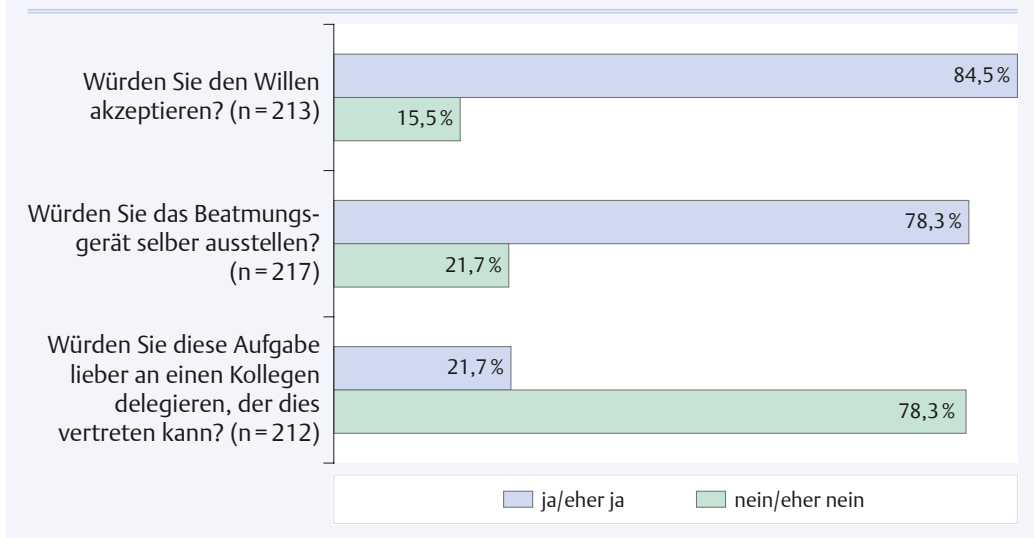

Abb. 2 Fallvignette zum persönlichen Umgang mit

erhoben, wobei sich die Befragung auf die Situation vor der Novellierung des Sterbehilfegesetzes im November 2015 bezieht ( $\downarrow$ Abb. 3). Daneben wurden die Wünsche der Intensivmediziner für die zukünftige Situation der PV erfasst ( $\bullet$ Abb. 4).

\section{Diskussion}

Im Rahmen dieser Studie wurden erstmals deutschlandweit die Sichtweisen leitender Intensivmediziner zum Umgang mit PV und der ärztlichen Handlungspraxis bezüglich therapiebegrenzender Maßnahmen am Lebensende untersucht. Die Ergebnisse bestätigen die Kontroverse der Einstellungen und Erfahrungen mit PV $[1,12,13]$ auch für den Bereich der Intensivmedizin. Trotz einer Vielzahl geäußerter Probleme wird die PV jedoch von mehr als zwei Drittel der Ärzte generell als hilfreich empfunden, wobei sich mehr als drei Viertel eine zusätzliche Vorsorgevollmacht wünschen. Nur jeder zwanzigste Arzt plädiert für eine Abschaffung der PV. Zukünftig benötigt es daher unterstützende Strategien und Konzepte zur wirksamen Umsetzung von PV [14].
Gesetzeslage zum ärztlich assistierten Suizid

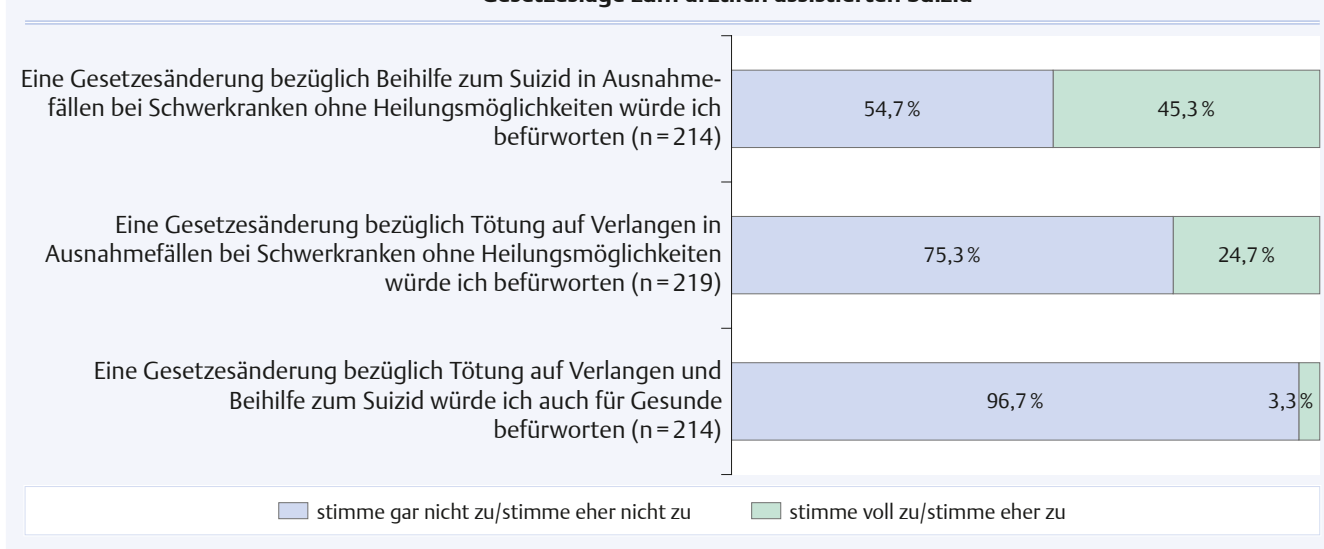

Abb. 3 Meinungsbild zur Gesetzeslage zum ärztlich assistierten Suizid. 
Zukünftig wünsche ich mir bezogen auf die Patientenverfügung (PV), ...

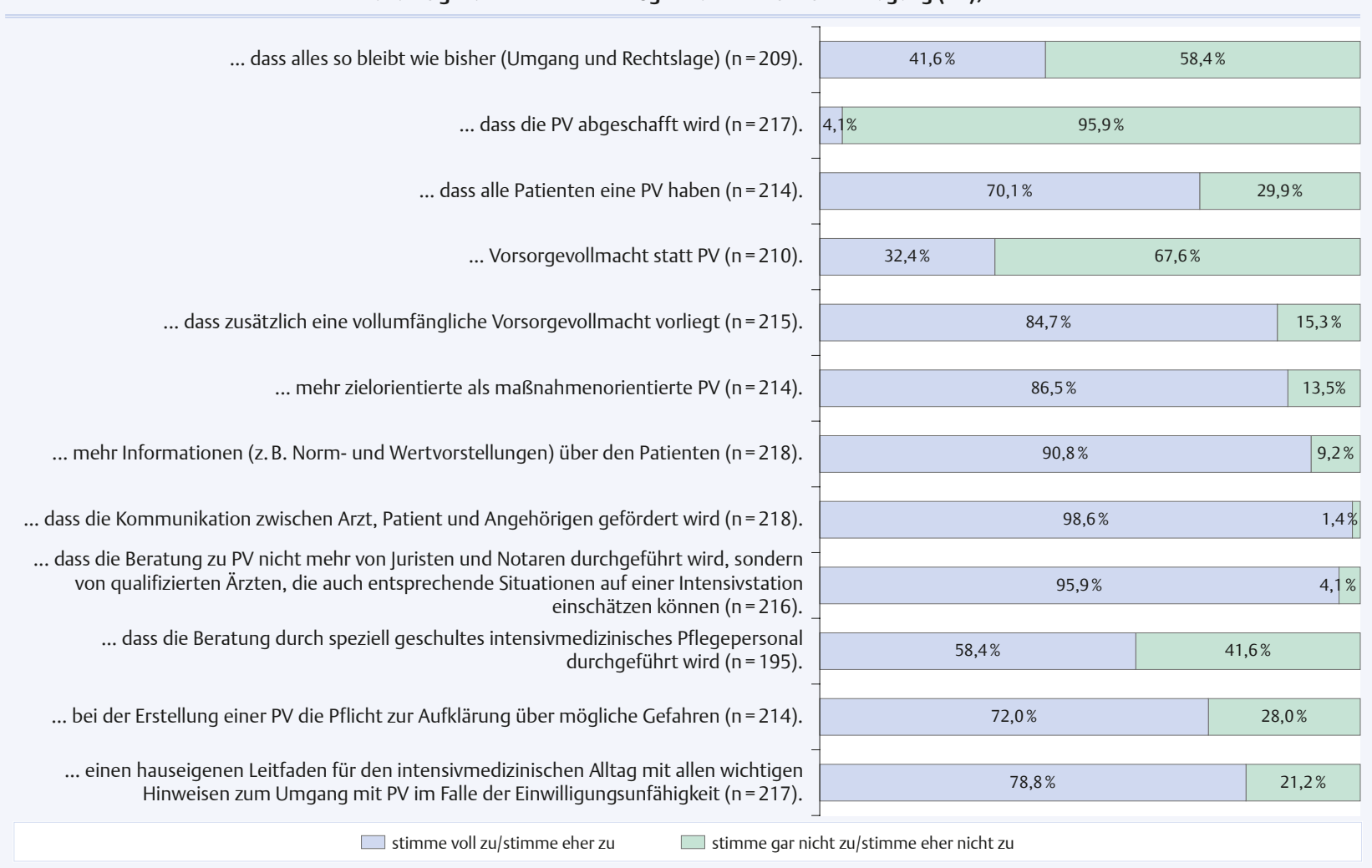

Abb. 4 Wünsche der Intensivmediziner für die zukünftige Situation der Patientenverfügung.
Probleme bei der Umsetzbarkeit | Insbesondere allgemeine oder pauschale Formulierungen und die geringe Kongruenz zur tatsächlich vorliegenden Krankheitssituation erschweren die Anwendbarkeit der PV im intensivmedizinischen Alltag. Ähnliche Ergebnisse zeigten sich auch in einer Untersuchung auf vier multidisziplinären Intensivstationen zur Beurteilung der Gültigkeit von PV durch Ärzte und Angehörige [3]. Diese bewerteten jede zweite PV unterschiedlich, wobei Intensivmediziner eine PV eher sinngemäß interpretierten, während Angehörige sie eher wortwörtlich auffassten. Auch untereinander stimmten die Ärzte nur in der Hälfte der Fälle überein [3]. In einer österreichischen Erhebung eines Universitätskrankenhauses wurden Unsicherheiten über die Gültigkeit von PV ebenso bei Intensivpflegekräften thematisiert [15]. Dementsprechend wird die Empfehlung die PV mehr an Therapiezielen statt an Maßnahmen auszurichten [1] in der vorliegenden Studie von der Mehrheit der Intensivmediziner befürwortet.

Als unterstützende Maßnahme im Umgang mit Entscheidungen über eine Therapiezieländerung bei schwerkranken und sterbenden Patienten, inklusive des Umgangs mit PV, wurde im Rahmen einer Prä-Post-Studie eine Klinik-Leitlinie für Ärzte und Pflegende auf Intensivstationen evaluiert [6]. Diese führte zu größerer Handlungssicherheit bei den Mitarbeitern, insbesondere aber nahm die Angst vor Rechtsfolgen ab. Auch drei Viertel der Befragungsteilnehmer wünschen sich einen hauseigenen Leitfaden für den intensivmedizinischen Alltag zum Umgang mit der PV im Falle der Einwilligungsunfähigkeit.

Aussagekräftige Patientenverfügungen | Drei Viertel der befragten Intensivmediziner haben die Erfahrung gemacht, Patienten oder Angehörige über die inhaltliche Bedeutung von in der PV getroffenen Aussagen in Bezug auf die akute klinische Situation erst aufklären zu müssen. Insofern plädiert die Mehrzahl der Befragten für eine fachliche Beratung zu (intensiv-)medizinischen Inhalten einer PV durch entsprechend qualifizierte Ärzte; die Hälfte kann sich eine Beratung außerdem durch speziell geschulte Intensivpflegekräfte vorstellen. Den positiven Einfluss solcher fachlicher Beratung auf die inhaltliche Gestaltung von PV verdeutlichen Evaluationsergebnisse von Patientenseminaren zur Thematik [16]. Fast alle Seminarteilnehmer mit einer vorab ohne Beratung in medizinischen Fragen erstellten PV sahen nach dem Beratungsseminar erheblichen Korrektur- oder Konkretisierungsbedarf ihrer PV.

Eine Chance zur Erstellung aussagekräftiger PV bietet darüber hinaus das international bereits viel beachtete Konzept „Advance Care Planning“ $[17,18]$. Zentral ist dabei eine Vorausplanung künftiger medizinischer und pflegerischer Behandlungsentscheidungen, die im Gesprächsprozess zwischen dem Betroffenen, seinem Angehörigen (beziehungsweise Vertreter) und speziell geschultem Fachpersonal gemeinsam entwickelt 
wird [17]. Jedoch bestätigt die vorliegende Untersuchung, dass ACP als Beratungsangebot zur Versorgungsplanung in der Intensivmedizin in Deutschland noch weitgehend unbekannt ist.

Stärken und Limitationen der Studie I Die Erhebung erfolgte als postalische Befragung mit der Gefahr einer eingeschränkten Repräsentativität durch einen geringen Rücklauf der Fragebögen. Die erzielte Rücklauf-Rate von 74,2\% liegt hingegen deutlich über den Rücklaufquoten vergleichbarer Befragungen mit ca. 40\% [19, 20]). Zudem ergab die Non-Responder-Analyse keinen Hinweis auf eine systematische Nichtteilnahme. Die Ergebnisse sind jedoch limitiert auf die Stichprobe von Entscheidungsträgern anästhesiologisch geführter Intensivstationen in Kliniken mit mindestens 300 Betten. Diese Begrenzung resultierte aus der Annahme, dass in kleineren Kliniken aufgrund der geringer ausgeprägten Fallschwere der versorgten Patienten Patientenverfügungen hinsichtlich unserer Fragestellung eine eher untergeordnete Rolle spielen. Durch die Eingrenzung auf anästhesiologisch geführte Intensivstationen sollte vermieden werden, verschiedene Fachrichtungen mit unterschiedlichen Behandlungsstrategien miteinander zu bewerten. Da studien-

\section{Literatur}

1 Jox RJ, in der Schmitten J, Marckmann G. Ethische Grenzen und Defizite der Patientenverfügung. In: Coors M, Jox RJ, in der Schmitten J, Hrsg. Advance Care Planning. Stuttgart: W. Kohlhammer; 2015: 23-38

2 Schick G. Patientenverfügung in der Intensiv- und Notfallmedizin. In: May AT, Kreß H, Verrel T, Wagner T, Hrsg. Patientenverfügungen. Berlin, Heidelberg: Springer; 2016: 177-188

3 Leder N, Schwarzkopf D, Reinhart K et al. The validity of advance directives in acute situations. Dtsch Arztebl Int 2015; 112: 723-729

4 Hartog CS, Peschel I, Schwarzkopf D et al. Are written advance directives helpful to guide end-oflife therapy in the intensive care unit? A retrospective matched-cohort study. J Crit Care 2014; 29: 128-133

5 Riessen R, Bantlin C, Wiesing U, Haap M. Therapiezieländerungen auf einer internistischen Intensivstation. Einfluss von Willensäußerungen der Patienten auf Therapieentscheidungen. Med Klin Intensivmed Notfmed 2013; 108: 412-418

6 Jox RJ, Winkler EC, Borasio GD. Änderung des Therapieziels am Lebensende: Effekte einer KlinikLeitlinie. Dtsch Med Wochenschr 2012; 137: 829-833

7 Graw JA, Spies CD, Wernecke KD, Braun JP. Managing end-of-life decision making in intensive care medicine: a perspective from Charite Hospital, Germany. PLoS One 2012; 7: e46446

8 Dillman DA. Mail and telephone surveys: the total design method. New York: Wiley; 1978

9 Langer S, Knorr J-U, Berg A. Umgang mit Patientenverfügungen: Probleme durch pauschale Formulierungen. Dtsch Arztebl 2013; 110: A-2186/B-1924/C-870

10 Prüfer P, Rexrodt M. Zwei-Phasen-Pretesting: ZUMA-Arbeitsbericht 2000/08; 2000 methodisch keine fall- oder verlaufsbezogene Datenerfassung geplant war, beruht die Untersuchung ausschließlich auf der subjektiven Einschätzung und dem Meinungsbild der leitenden Intensivmediziner zum Erhebungszeitpunkt.

\section{Konsequenz für Klinik und Praxis}

- Trotz einer Vielzahl von Problemen im Umgang mit Patientenverfügungen empfinden mehr als zwei Drittel der Intensivmediziner sie generell als hilfreich.

- Die Mehrzahl der Ärzte plädiert für mehr auf das Therapieziel orientierte und weniger auf Maßnahmen orientierte Patientenverfügungen.

- Patienten brauchen eine Beratung zu intensivmedizinischen Inhalten durch fachlich qualifiziertes Personal.

- Finale Extubation gemäß dem Patientenwillen wird in acht von zehn Kliniken durchgeführt; jeder zweite Arzt erlebt den Vorgang der finalen Extubation als belastend.

- Unterstützende Konzepte und Maßnahmen zur Erstellung aussagekräftiger Patientenverfügungen und einer wirksamen Umsetzung sind notwendig.

http://www.gesis.org/fileadmin/upload/forschung/ publikationen/gesis_reihen/zuma_ arbeitsberichte/00_08.pdf Letzter Zugriff am 29.02.2016

11 Bundesgerichtshof. Urteil vom 25.06.2010; 2 StR 454/09. BGHSt 55, 191

12 Kierig FO, Behlau W. Der Wille des Patienten entscheidet: Patientenverfügung, Vorsorgevollmacht und Behandlungsabbruch. Heidelberg: C. F. Müller; 2011

13 Klinkhammer G. Pro und Kontra: Patientenverfügungen. Dtsch Arztebl 2015; 112: A-716/B-606/C-586

14 in der Schmitten J, Marckmann G. Gesundheitliche Vorausplanung (Advance Care Planning): Was können wir aus internationalen Erfahrungen für die Umsetzung von Patientenverfügungen lernen? In: Borasio GD, Heßler H-J, Jox RJ, Meier C, Hrsg. Patientenverfügung: Das neue Gesetz in der Praxis. Stuttgart: W. Kohlhammer; 2012: 96-114

15 Heindl P, Ruppert S, Kozon V. Umgang von österreichischen Pflegepersonen mit der Patientenverfügung. Pflege 2014; 27: 337-346

16 Schöffner M, Schmidt KW, Benzenhöfer U, Sahm S. Patientenverfügung auf dem Prüfstand: Ärztliche Beratung ist unerlässlich. Dtsch Med Wochenschr 2012; 137: 487-490

17 Coors M, Jox RJ, in der Schmitten J: Advance Care Planning: Von der Patientenverfügung zur gesundheitlichen Vorausplanung. Stuttgart: W. Kohlhammer; 2015

18 Meyer G, Köpke S. Advanced Care Planning ist umsetzbar. Vorausplanung der Pflege und Versorgung am Lebensende. Pflege Z 2014; 67: 404-406

19 Wandrowski J, Schuster T, Strube W, Steger F. Medical ethical knowledge and moral attitudes among physicians in Bavaria. Dtsch Arztebl Int 2012; 109: 141-147

20 Schildmann J, Dahmen B, Vollmann J. Ärztliche Handlungspraxis am Lebensende. Dtsch Med Wochenschr 2015; 140: e1-e6
Interessenkonflikt

Die Autoren geben an, dass kein Interessenkonflikt besteht.

DOI 10.1055/s-0042-104038 Dtsch Med Wochenschr 2016; 141: e73-e79 (c) Georg Thieme Verlag KG . Stuttgart · New York . ISSN 0012-0472 